www.jmscr.igmpublication.org

Index Copernicus Value: 79.54

ISSN (e)-2347-176x ISSN (p) 2455-0450

crossrefDOI: https://dx.doi.org/10.18535/jmscr/v7i2.175

\title{
Prospective Randomized Study to Compare the Motor Recovery with Epidural Analgesia using 0.2\% Ropivacaine and 0.175\% Bupivacaine in Abdominal Surgery
}

\author{
Authors \\ S. R. Karthick ${ }^{1}$, Satish Logidasan ${ }^{2}$, Kanimozhi $\mathbf{R}^{3}$, Arulraj G.P ${ }^{4}$, Karthik.A ${ }^{5}$ \\ S Prasana Vathanan ${ }^{6}$, Ambal $\mathrm{S}^{7}, \mathrm{C} \mathrm{Sathish}^{8}$ \\ Corresponding Author \\ A Gowri Shankar \\ Department of Anesthesiology, Govt Stanley Medical College, TN- 600001, India \\ Email:agsmbbs@gmail.com
}

\begin{abstract}
A combination of $0.2 \%$ ropivacaine with $2 \mu \mathrm{g} / \mathrm{ml}$ fentanyl can be very well used as postoperative analgesic regimen in major abdominal surgeries as it provides a clear balance between analgesia and motor blockade.In this prospective randomised comparative study conducted in 100 patients, the postoperative pain relief and motor recovery with epidural analgesia using $0.2 \%$ ropivacaine with fentanyl and $0.175 \%$ bupivacaine with fentanyl in major abdominal surgeries were compared.

Group R: Patients receiving ropivacaine and fentanyl epidurally

Group B: Patients receiving bupivacaine and fentanyl epidurally

$0.2 \%$ Ropivacaine with fentanyl group offers good postoperative pain relief comparable to Bupivacine and better motor recovery than $0.175 \%$ Bupivacaine in epidural analgesia. No adverse reactions and complications were noted. Secondary outcomes like Postoperative Nausea and vomitting, Rescue Analgesic requirement, Patient Satisfaction, Occurence of side effects were comparable in both the groups.

Keywords: Ropivacaine, Epidural analgesia, postoperative pain relief.
\end{abstract}

\section{Introduction}

Advances in perioperative anaesthesia and analgesia has improved pain relief, patient satisfaction and early mobilization in abdominal surgeries ${ }^{[1]}$. Ropivacaine is generally cardiostable when compared with bupivacaine and preferentially blocks ' $\mathrm{C}$ ' fibres better than A fibres. Thus ropivacaine produces less ' $A$ ' fibre blockade with proportional concentration of bupivacaine, whereas 'C' fibre blockade was similar with both drugs ${ }^{[2]}$. This unique property of ropivacaine provides analgesia with lesser motor blockade than comparable concentrations of bupivacaine. Lipophilic opioids like fentanyl and sufentanyl diffuse rapidly across the dura and arachnoid matter and produces effective analgesia on comparison with hydrophilic opioids ${ }^{[3]}$. In this prospective randomised controlled study we had compared the pain relief and mobilization during epidural anaesthesia with ropivacaine $0.2 \%$ compared with bupivacaine $0.175 \%$ in combination with $2 \mu \mathrm{g} / \mathrm{ml}$ of the lipophylic opioid fentanyl. 


\section{Aim}

To compare the postoperative pain relief and motor recovery with epidural analgesia using $0.2 \%$ ropivacaine with fentanyl and $0.175 \%$ bupivacaine with fentanyl in major abdominal surgeries.

\section{Materials and Methods}

All the 100 patients were allocated to either the ropivacaine fentanyl group (Group R) or bupivacaine fentanyl group (Group B) by sealed cover technique. Study was a single blinded study. The patients who met the inclusion and exclusion criteria were only included in the study. Patients were divided into 2 groups of 50 each.

Group R: Patients receiving ropivacaine and fentanyl epidurally

Group B: Patients receiving bupivacaine and fentanyl epidurally

\section{Inclusion Criteria}

All consented patients with

Age: $18-65$ years

Both genders

Weight: $>=45 \mathrm{Kg}$

ASA: $1,2 \& 3$

Elective major abdominal surgery

\section{Exclusion Criteria}

1. Patients with impaired kidney or Liver functions

2. History of alcohol abuse, History of chronic pain, Daily intake of analgesics

3. Patients with infection at the site of epidural injection

4. Patients on Anti - coagulant therapy

5. Patients with severe Aortic/ Mitral stenosis

6. Patients allergic to local anaesthetics

\section{Procedure}

Intravenous access - 18 gauge IV line was secured and ringer lactate was started at $2 \mathrm{ml} / \mathrm{kg} / \mathrm{hr}$

Premedication: Injection midazolam $0.02 \mathrm{mg} / \mathrm{kg} \mathrm{mg}$, Injection glycopyrolate $4 \mu \mathrm{G} / \mathrm{kg}$, Injection Fentanyl $2 \mu \mathrm{g} / \mathrm{kg}$ given through intravenous route.

Epidural catheter insertion: Under strict aseptic precautions, epidural thoracic catheters were inserted for postoperative pain control at T9 to T10 level before induction. Epidural test dose comprising $3 \mathrm{ml}$ of $1.5 \%$ lignocaine and $15 \mu \mathrm{g}$ of adrenaline was given.

Induction was done with thiopentone $5 \mathrm{mg} / \mathrm{kg}$ and endotracheal tube intubation facilitated with suxamethonium $2 \mathrm{mg} / \mathrm{kg}$. Anesthesia was maintained with desflurane $3 \%$ and $\mathrm{O} 2 / \mathrm{N} 2 \mathrm{O}$ mixture with a fraction of inspired O2 0.4. Muscle relaxation was maintained with Injection Vecuronium $0.1 \mathrm{mg} / \mathrm{kg}$ followed by intermittent doses of $0.02 \mathrm{mg} / \mathrm{kg}$. Injection fentanyl $25 \mu \mathrm{g}$ bolus was administered intravenously for every $20 \%$ rise in heart rate and Mean arterial pressure(MAP) from the baseline value.

At the end of surgery patients were reversed with Neostigmine $0.5 \mathrm{mg} / \mathrm{kg}$ and glycopyrrolate 0.08 $\mathrm{mg} / \mathrm{kg}$ and then extubated. All the patients were shifted to post-anesthetic care unit (PACU) for the first 48 hours. Epidural infusion was initiated providing a continuous delivery of ropivacaine $0.2 \%$ and $2 \mu \mathrm{g} / \mathrm{ml}$ of fentanyl for the group $\mathrm{R}$ and bupivacaine $0.175 \%$ and $2 \mu \mathrm{g} / \mathrm{ml}$ of fentanyl for the group $\mathrm{B}$ at the rate of $5 \mathrm{ml} /$ hour for 48 hours postoperatively.

At Immediate, 30 minutes, 60 minutes, 2nd hour, 6th hour, 12th hour, 18th hour, 24th hour, 30 th hour, 36 th hour and 48th hour following surgery, level of postoperative pain was assessed using Visual Analogue Scale (VAS) (starting from 0-no pain to 10 -worst pain imaginable). Injection tramadol $100 \mathrm{mg}$ intramuscularly administered for patients with a score more than 4 as rescue analgesic after giving $4 \mathrm{mg}$ injection ondonsetron intravenously. The number of patients who received rescue analgesic drug were noted. MAP was measured over the entire 48 hours postoperative period, at specified time intervals. The mean arterial pressure was found to be comparable in both the groups at all time intervals. The $\mathrm{P}$ - value was found to be statistically insignificant, at all time intervals. Postoperative mobilization was encouraged $6 \mathrm{hrs}$ after shifting to PACU. Postoperative ambulatory score was assessed at 6hrs, 12 hrs, 24 hrs, 36 hrs, 48 hrs, 60 hrs, 72hrs, 84 hrs, 
96 hrs postoperatively. All data were recorded by residents of anesthesia not participated in the study.

\section{Hemodynamics Management}

Hemodynamic parameters were monitored for 48 hrs at the following intervals: Immediate, 30 minutes, 60 minutes, 2nd hour, 6th hour, 12th hour, 18th hour, 24th hour, 30 th hour, 36 th hour and 48th hour following surgery.

Hypotension was defined as mean arterial pressure $<20 \%$ from baseline parameters. Episodes of hypotension were treated with fluid boluses of normal saline or ringer lactate. Patients not responding to crystalloids were given injection ephedrine intravenously Complications like bradycardia, respiratory depression if any were also recorded.

\section{Primary Outcome measures}

1. Assessment of the postoperative analgesia by Visual Analogue pain score.

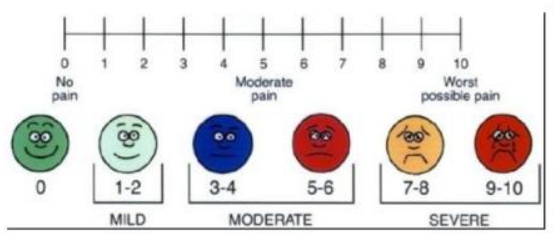

Fig 1. VAS

2.Assesment of postoperative mobilization

\begin{tabular}{|l|l|}
\hline SCORE & PATIENT AMBULATION \\
\hline 1 & Lying in bed \\
\hline 2 & Able to sit outside the bed \\
\hline 3 & $\begin{array}{l}\text { Able to move outside the bed for } \\
\text { limited time ( }<30 \mathrm{~min})\end{array}$ \\
\hline 4 & Able to move without limits \\
\hline
\end{tabular}

Fig 2 Ambulation score

Secondary Outcome measures:

Postoperative Nausea and vomitting

Rescue Analgesic requirement

Patient Satisfaction

Occurence of side effects

Patients were assessed for nausea and vomiting by the following score

None $=0$

Mild $=1$

Moderate $=2$
Vomiting $=3$

Rescue antiemetics were given to patients with nausea score greater than are equal to 2 with Injection Ondansetron 4mg

b. Patient Satisfaction:

Patient satisfaction was assessed at the end of 48 hours.

$$
\begin{aligned}
& \text { Poor }=1 \\
& \text { Fair }=2 \\
& \text { Good }=3 \\
& \text { Excellent }=4
\end{aligned}
$$

c. Therapeutic failure is defined as inadequate pain relief from surgical wound and drains.

d. Technical Failure is the inability to insert epidural catheter as a result of poor tissue planes.

Requirement of rescue analgesia: If the postoperative pain scores as measured by visual analogue scale is greater than or equal to 4 , then rescue analgesia was given with intravenous tramadol 100mg. Complications were monitored for 48 hours.

\section{Observations and Results}

The mean VAS scores at all the time intervals measured were comparable between both the groups. The $p$ value computed was statistically insignificant. So the analgesic efficacy of both groups ' $R$ ' and ' $B$ ' were comparable.

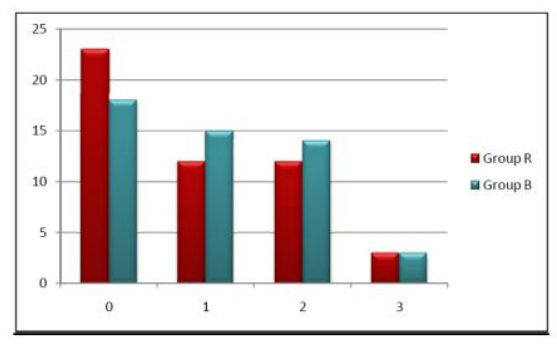

Fig 3 VAS

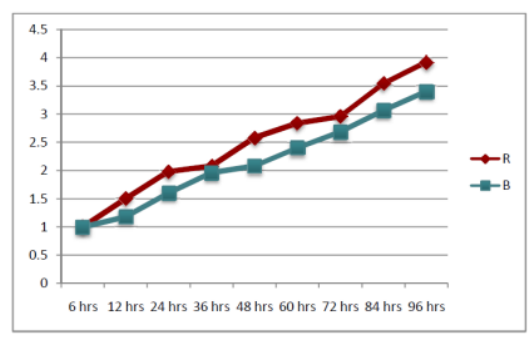

Fig 4 Ambulation score 
Fig4. Postopeartive mobilization was assessed by postoperative ambulation score at the intervals of 6hrs, 12 hrs, 24 hrs, 36 hrs, 48 hrs, 60 hrs, 72 hrs, $84 \mathrm{hrs}$ and $96 \mathrm{hrs}$. The mean postoperative ambulation scores were significant during all the time intervals except at $6 \mathrm{hrs}$ where it is statistically insignificant. The scores were highly significant during 12 hrs, 24 hrs, 48hrs, 60 hrs, 72 hrs, 84 hrs and $96 \mathrm{hrs}$.

The therapeutic failure rates were comparable between both the groups, with the $\mathrm{p}$ value being 0.646. Out of the five patients who underwent failure, three patients had block in epidural catheter and two patients had bloody aspirates postoperatively. In all the patients who had failure, rescue analgesia was given.

One of the secondary outcome measures that was analyzed was the postoperative nausea and vomiting. Rescue antiemetics were given with injection Ondansetron, $4 \mathrm{mg}$ intravenously, when PONV scores were $\geq 2$. Average PONV scores were similar in both the groups.

\section{Discussion}

Though the administration of epidural analgesia alleviates pain, one of its major limitation is the motor blockade caused by the local anaesthetic used which hinders the early postoperative mobilization $^{[4]}$.

It has been established that ropivacaine which is similar in structure to that of commonly used local anaesthetic bupivacaine, has lesser propensity to cause motor blockade, especially when used on a $\mathrm{mg} / \mathrm{mg}$ basis $^{[5]}$. In addition as discussed earlier addition of lipophilic opioids to either group increases the quality of analgesia due to synergism. Previously a dose finding study for postoperative analgesia was done by Scott DA and colleagues concluded that $0.2 \%$ ropivacaine was the essential dose of ropivacaine that provides optimum balance between analgesia and motor block ${ }^{[6]}$. We compared the pain scores between 2 groups over the $48 \mathrm{hrs}$ period postoperatively and postoperative ambulation score for 4 postoperative days. Hudgson PS, Liu SS have done a study, A comparison of ropivacaine to bupivacaine with fentanyl for post operative patient controlled epidural analgesia.

A sum of 40 patients undergoing abdominal surgery underwent a randomized double blinded study to compare the following: $0.05 \%$ bupivacaine/ fentanyl, $0.1 \%$ bupivacaine/fentanyl, $0.05 \%$ ropivacaine /fentanyl or $0.1 \%$ ropivacaine/ fentanyl for standardized PCEA. They measured pain scores, adverse effects, and PCEA consumption for $42 \mathrm{~h}$.

Lower extremity motor function was assessed. Analgesia was similar among both the groups. Local anaesthetic drug consumption was a bit higher in the $0.1 \%$ bupivacaine than that of $0.1 \%$ ropivacaine groups. Motor function decreased a fraction again similar among the groups. Eight patients were temporarily unable to ambulate during the study. These patients consumed higher fraction of local anaesthetic drugs with additional decrease in motor function compared with other ambulating patients. In addition postoperative nausea and vomiting, patient satisfaction, rescue analgesia with injection tramadol, complications associated with the procedure were evaluated between the 2 groups. The hemodynamic parameters over a period of 48 hours were also compared between both the groups. Epidural catheters were placed before induction of anaesthesia in both the groups. Epidural analgesia was activated immediately after shifting the patient to post-anaesthesia care units in both the groups. The motor block minimal local analgesic concentration (MMLC) for bupivacaine was found to be $0.326 \%$ and for ropivacaine was $0.497 \%^{[7]}$. The ropivacaine / bupivacaine potency ratio was 0.66 . This was the first MLAC study to determine and estimate the motor blocking potencies of bupivacaine and ropivacaine. It was concluded that ropivacaine almost always causes lesser motor blockade than that of the drug bupivacaine.

The next outcome measure was postoperative satisfaction score. A score of 4 means excellent postoperative satisfaction recorded in 17 patients in group ' $R$ ' and 12 patients in group ' $B$ '. The values were again comparable.

The therapeutic failure rates were comparable between both the groups ${ }^{[8]}$ Out of the five patients 
who underwent failure, three patients had block in epidural catheter and two patients had bloody aspirates postoperatively. In all the patients who had failure, rescue analgesia was given. There was no incidence of bradycardia, respiratory depression, urinary retention in both the groups. But there was recorded hypotension in both the groups. 5 of the 50 patients from Group $\mathrm{R}$ and 7 of the 50 patients from Group B had hypotension that is defined as mean arterial pressure $<20 \%$ from baseline parameters. Episodes of hypotension were treated with fluid boluses of normal saline or ringer lactate. Patients not responding to crystalloids were to be given injection ephedrine intravenously. But all patients responded to fluid boluses. Physiological effect of sympathetic blockade ${ }^{[9]}$, was reason behind this hypotension in both the groups. The incidence of hypotension was comparable between both the groups. As far as the hemodynamic parameters are concerned there was a fall in systolic blood pressure, as well as mean arterial pressure at periodic time intervals after activation of epidural catheter in both the groups. Rest of the parameters were comparable

\section{Conclusion}

This randomized controlled study conducted to compare the analgesic efficacy and ability to ambulate with postoperative epidural analgesia using $0.2 \%$ ropivacaine and fentanyl and $0.175 \%$ bupivacaine and fentanyl concluded that the level of postoperative analgesia is comparable in both the groups, with significantly better postoperative ambulation in the ropivacaine group. Thus, a combination of $0.2 \%$ ropivacaine with $2 \mu \mathrm{g} / \mathrm{ml}$ fentanyl can be very well used as postoperative analgesic regimen in major abdominal surgeries as it provides a clear balance between analgesia and motor blockade.

\section{References}

1. Role of epidural anaesthesia and analgesia in surgical practice - Robert J. Moraca,MD, David G Sheldon MD, Richard C MD.

2. Gerhard Brodner, MD, Phd, Novbelt, MD, Hufovan Aken, MD, PhD, Esther Pogatzler
MD Anaesthesia Analgesia 1999, 88; 128 33

3. Adjuvant agents in neuraxial blockade, ATOTW 230; 4 th July 2011 Dr. Nickole Khangure,

4. H. Kehlet and K. Holte et al BJA 2001; 8J; $47-61$; effect of postoperative analgesia on surgical outcome

5. Kehlet H, Mogensen $\mathrm{T}$, Hospital stay of 2days after open sigmoidectomy with a multimodal rehabilitation programme

6. Scott DA, Chamley DM, Mooney PH, Deam RK, Meak AH, Hafflof B, Epidural ropivacaine infusion for postoperative analgesia after major lower abdominal surge

7. Lacassie et al comparative abilities of the drugs ropivacaine and bupivacaine to cause a motor block in labor was assessed.

8. Bariskaner H, Ayaz M, Guney FB, Dalkilic $\mathrm{N}$, Guney O. Bupivacaine and ropivacaine : comparative effects on nerve conduction blockade

9. Liu SS, Moore JM, Luo AM, et al: Comparision of three solutions of ropivacaine /fentanyl for post operative patient controlled epidural analgesia. Anaesthesiology 90: 727, 1999. 